\title{
Shortened Activated Partial Thromboplastin Time May Help in Diagnosing Hereditary and Acquired Angioedema
}

\author{
Konrad Bork Günther Witzke \\ Department of Dermatology, Johannes Gutenberg University, Mainz, Germany
}

\section{Key Words}

Angioedema $\cdot \mathrm{C} 1$ inhibitor deficiency - Activated partial thromboplastin time · Factor XI - Thrombin-antithrombin complex with normal C1-INH and also to HP. Conclusions: A shortened APTT may help to diagnose HAE-C1-INH and AAE-C1$\mathrm{INH}$ when determination of $\mathrm{C1} 1 \mathrm{INH}$ is not yet available.

ㄷ) 2016 S. Karger AG, Basel

\section{Introduction}

Hereditary angioedema (HAE) is a rare disease characterized by recurrent angioedema attacks involving the skin, gastrointestinal tract and larynx. Laryngeal attacks are potentially life-threatening. To date, several different HAE types have been reported. HAE with $\mathrm{C} 1$-esterase inhibitor (C1-INH) deficiency, i.e. HAE-C1-INH, is associated with an inherited functional deficiency of C1-INH. In HAE-C1-INH type I, the C1-INH activity and protein concentration in the plasma are low. In HAE-C1-INH type II, the C1-INH activity is low but the protein is normal or even elevated. HAE with normal $\mathrm{C} 1-\mathrm{INH}$ (HAEnCI) or 'HAE type III' presents with normal or subnormal C1-INH activity in the plasma. In some families, patients and symptom-free family members have shown mutations in the F12 gene which affect the proline-rich region of the coagulation factor (F)XII protein, i.e. they have a normal C1-INH and an HAE-specific mutation in

\section{KARGER}

E-Mail karger@karger.com

www.karger.com/iaa
(C) 2016 S. Karger AG, Basel

1018-2438/16/1702-0101\$39.50/0
Correspondence to: Prof. Dr. Konrad Bork

Department of Dermatology, Johannes Gutenberg University Langenbeckstrasse 1

DE-55131 Mainz (Germany)

E-Mail bork@ hautklinik.klinik.uni-mainz.de 


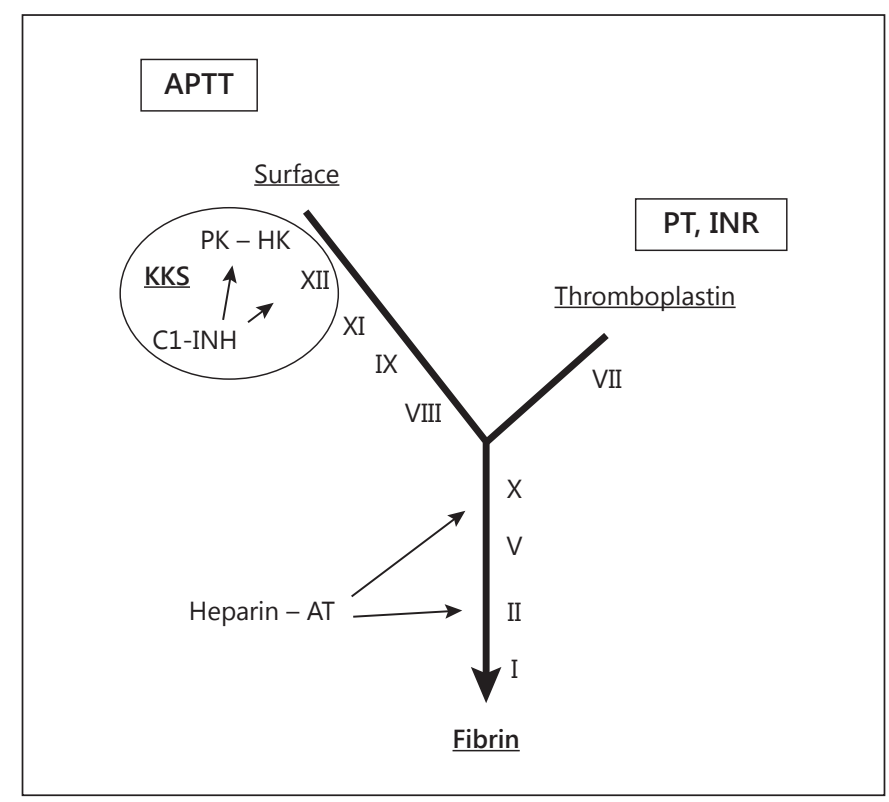

Fig. 1. Global coagulation tests. APTT and PT are in vitro laboratory screening tests that characterize the 'intrinsic (endogenous)', 'extrinsic (exogenous)' and the 'common' pathways of plasmatic blood coagulation. The intrinsic pathway also includes the KKS. For the determinations citrated PPP is used. PT can be used to calculate the INR. By a modification using deficient plasma, APTT and PT can be used to measure single coagulation factors. PK = Prekallikrein; $\mathrm{HK}$ = high molecular weight kininogen.

the F12 gene (HAE-FXII) [1]. However, to date, no mutations have been identified for the remaining types of HAEnCI, i.e. those with no HAE-specific mutations in the F12 gene (HAE-unknown). Apart from the hereditary form of angioedema, various acquired forms also exist. Acquired angioedema (AAE) with C1-INH deficiency, i.e. AAE-C1-INH, is associated with low $\mathrm{C} 1-\mathrm{INH}$ activity and protein concentration in the plasma whereas these are normal in the histaminergic $(\mathrm{h})$ and nonhistaminergic (nh) forms of so-called idiopathic angioedema (IAE), i.e. IAE-h and IAE-nh, respectively. It is assumed that the major mediator of HAE-C1-INH and AAE-C1-INH attacks is bradykinin $[2,3]$.

The diagnosis of HAE-C1-INH is based on the presentation of recurrent, localized subcutaneous or submucosal edema lasting for 2-5 days, a positive family history in most cases and a laboratory confirmation. Patients with HAE-C1-INH and AAE-C1-INH have C1-INH activity of between 0 and $50 \%$ and low concentrations of complement component $4(\mathrm{C} 4)$. The plasma reference (normal) range (RR) for C1-INH activity is between 70 and $130 \%$, and between 16.4 and $31.3 \mathrm{mg} / \mathrm{dl}$ for $\mathrm{C} 4$ protein concentration. In patients with HAEnCI, C1-INH activity is normal or subnormal and C4 is normal [4].

In a previous study, we showed that patients with HAE-C1-INH type I had markedly elevated clotting activities of FXII, FXI and FV and a shorter activated partial thromboplastin time (APTT) when compared with healthy participants (HP) [5]. APTT is an in vitro laboratory screening test that characterizes the intrinsic (endogenous)' and 'common' pathways of plasmatic blood coagulation. The test also includes the kallikrein-kinin system (KKS) (fig. 1). Our results demonstrated an activation of the kallikrein-kinin (contact) and the intrinsic coagulation system in patients with HAE-C1-INH. Similar results were obtained in other studies, including the formation of complexes of C1-INH with target proteases and an increased formation of thrombin-antithrombin (TAT) complexes and prothrombin fragments 1 and 2 [6-16].

C1-INH and C4 determinations are not available in all hospitals and normally not on a routine basis. The results are usually only obtained a few days or weeks later. In contrast, the determination of APTT is routine, easy to perform and available in most hospitals. Thus, the APTT value may be one of the first available laboratory results.

Based on our observation of a shortening of APTT in patients with HAE-C1-INH, the aim of this prospective study was to identify if APTT was not shortened in angioedema with normal C1-INH including HAEnCI, IAE-h and IAE-nh, and that, were this the case, whether APPT could be a useful tool for the early diagnosis of HAE-C1$\mathrm{INH}$ and also serve to differentiate it from other forms of recurrent angioedema.

\section{Material and Methods}

\section{Subjects}

For this prospective study, a total of 149 adult patients with various types of $\mathrm{HAE}$ and $\mathrm{AAE}$ and $26 \mathrm{HP}$ from the angioedema outpatient service of the Department of Dermatology, Johannes Gutenberg University, Mainz, Germany, in 2012-2015, were analyzed. Inclusion criteria were recurrent angioedema without urticaria, no history of coagulation disorders and no anticoagulation by drugs. The separation of patients into the diagnostic groups identified was based on the final diagnosis. Criteria were: (1) for HAE-C1-INH, low C1-INH activity and onset of symptoms in childhood or adolescence; (2) for HAE-FXII, normal C1-INH activity and the presence of $1 \mathrm{HAE}$-specific mutation in exon 9 of the F12 gene; (3) for HAE-unknown, normal C1-INH activity, $\geq 2$ family members affected with recurrent angioedema and no $\mathrm{HAE}$ specific mutations in the F12 gene; (4) for AAE-C1-INH, low C1INH activity, onset of symptoms at the age of $\geq 40$ years, no angio- 
Table 1. Laboratory results (citrated plasma) of patients with various forms of angioedema during attack-free intervals and HP

\begin{tabular}{|c|c|c|c|c|c|c|c|c|}
\hline Diagnosis & $\begin{array}{l}\text { C1-INH } \\
\text { activity, \% }\end{array}$ & $\begin{array}{l}\mathrm{C} 1-\mathrm{INH} \\
\text { protein, } \\
\mathrm{mg} / \mathrm{dl}\end{array}$ & $\begin{array}{l}\text { C4 } \\
\text { protein, } \\
\text { mg/dl }\end{array}$ & APTT, s & INR & $\begin{array}{l}\text { FXI } \\
\text { clotting } \\
\text { activity, \% }\end{array}$ & $\begin{array}{l}\text { FXII } \\
\text { clotting } \\
\text { activity, \% }\end{array}$ & $\mathrm{TAT}, \mu \mathrm{g} / \mathrm{l}$ \\
\hline \multicolumn{9}{|l|}{$\mathrm{HP}(\mathrm{n}=26)$} \\
\hline Mean \pm SD & $94.1 \pm 17.5$ & $21.7 \pm 5.6$ & $21.5 \pm 8.7$ & $29.5 \pm 2.4$ & $0.97 \pm 0.07$ & $108.2 \pm 16.4$ & $113.2 \pm 28.9$ & $2.8 \pm 1.4$ \\
\hline \multicolumn{9}{|c|}{ HAE-C1-INH type I $(\mathrm{n}=41)$} \\
\hline Mean \pm SD & $13.5 \pm 12.8$ & $6.0 \pm 2.8$ & $6.6 \pm 3.1$ & $25.2 \pm 2.4$ & $0.98 \pm 0.09$ & $131.3 \pm 21.6$ & $127.8 \pm 25.5$ & $5.8 \pm 7.1$ \\
\hline $\mathrm{p}$ value & $<0.0001$ & $<0.0001$ & $<0.0001$ & $<0.0001$ & 0.5952 & $<0.0001$ & 0.0329 & 0.0418 \\
\hline \multicolumn{9}{|c|}{ HAE-C1-INH type II $(\mathrm{n}=4)$} \\
\hline Mean \pm SD & $10.6 \pm 19.1$ & $28 \pm 3.7$ & $4.8 \pm 0$ & $25.1 \pm 1.9$ & $0.95 \pm 0.06$ & $121.3 \pm 8.2$ & $114.2 \pm 38.4$ & $4.2 \pm 1.8$ \\
\hline $\mathrm{p}$ value & $<0.0001$ & 0.0389 & 0.007 & 0.0017 & 0.6231 & 0.1336 & 0.9498 & 0.1067 \\
\hline \multicolumn{9}{|c|}{ HAE-FXII $(n=13)$} \\
\hline Mean \pm SD & $98.7 \pm 16.6$ & $21.8 \pm 2.9$ & $22.8 \pm 5$ & $29.6 \pm 3.5$ & $0.95 \pm 0.09$ & $106.9 \pm 19.1$ & $102.1 \pm 23.2$ & $4.7 \pm 7.1$ \\
\hline $\mathrm{p}$ value & 0.4356 & 0.9357 & 0.6114 & 0.9361 & 0.5672 & 0.8252 & 0.2386 & 0.1949 \\
\hline \multicolumn{9}{|c|}{ HAE-unknown $(\mathrm{n}=14)$} \\
\hline Mean \pm SD & $96.8 \pm 17$ & $21.7 \pm 6.6$ & $27.6 \pm 7.6$ & $29.1 \pm 3.2$ & $0.98 \pm 0.08$ & $117.2 \pm 16.1$ & $107.6 \pm 30.7$ & $6.5 \pm 5.7$ \\
\hline $\mathrm{p}$ value & 0.6408 & 0.9976 & 0.0317 & 0.6461 & 0.7125 & 0.1041 & 0.5745 & 0.0031 \\
\hline \multicolumn{9}{|c|}{ AAE-C1-INH $(\mathrm{n}=15)$} \\
\hline Mean \pm SD & $10.5 \pm 11.4$ & $7.8 \pm 3.7$ & $4.8 \pm 0$ & $24.5 \pm 2.8$ & $0.94 \pm 0.05$ & $137 \pm 19.4$ & $132.7 \pm 31.5$ & $9.8 \pm 14.8$ \\
\hline $\mathrm{p}$ value & $<0.0001$ & $<0.0001$ & $<0.0001$ & $<0.0001$ & 0.1817 & $<0.0001$ & 0.0506 & 0.0212 \\
\hline \multicolumn{9}{|c|}{$\mathrm{IAE}-\mathrm{h}(\mathrm{n}=32)$} \\
\hline Mean \pm SD & $91.3 \pm 19$ & $21.8 \pm 4.2$ & $24.4 \pm 18.8$ & $30.2 \pm 2.4$ & $0.98 \pm 0.07$ & $103.3 \pm 12.4$ & $109.1 \pm 25.9$ & $4.1 \pm 3.3$ \\
\hline $\mathrm{p}$ value & 0.5625 & 0.9443 & 0.4669 & 0.3501 & 0.4347 & 0.1987 & 0.5706 & 0.0913 \\
\hline \multicolumn{9}{|c|}{ IAE-nh $(\mathrm{n}=30)$} \\
\hline Mean \pm SD & $99.9 \pm 23.7$ & $21.3 \pm 4.7$ & $25.1 \pm 10.3$ & $28.5 \pm 2.5$ & $0.96 \pm 0.06$ & $124.5 \pm 18.2$ & $117.4 \pm 21.5$ & $3.6 \pm 1.98$ \\
\hline $\mathrm{p}$ value & 0.3013 & 0.7987 & 0.1619 & 0.1077 & 0.6128 & 0.001 & 0.5351 & 0.1050 \\
\hline $\mathrm{RR}$ & $70-130$ & $15.4-33.8$ & $16.4-31.3$ & $26-36$ & $0.8-1.2$ & $70-120$ & $70-150$ & $1-4.1$ \\
\hline
\end{tabular}

Lower detection limit of C1-INH and C4 protein $=4.8 \mathrm{mg} / \mathrm{dl}$; lower detection limit of C1-INH activity $=1 \%$.

edema in family members and, in some patients, underlying B cell disorder and/or low C1q; (5) for IAE-h, angioedema responsive to antihistamines with recurrence prevented by consistent use of second-generation antihistamines; (6) for IAE-nh, angioedema recurrences not prevented by consistent use of antihistamines, no other family members affected, normal C1-INH and no angioedema-specific mutation in the F12 gene.

\section{Laboratory Methods}

Blood samples were collected by careful peripheral venipuncture into $0.106 \mathrm{~mol} / \mathrm{l}$ trisodium citrate solution $(1 \mathrm{ml}$ anticoagulant: $9 \mathrm{ml}$ whole blood; S-Monovette tubes, Sarstedt AG, Nümbrecht, Germany). As soon as possible, the citrated blood was centrifuged at $1,500 \mathrm{~g}$ for $10 \mathrm{~min}$ at room temperature and the platelet-poor plasma (PPP) was drawn off. It was used immediately for the determination of APTT and prothrombin time (PT). In case further determinations were not yet possible, the PPP was aliquoted and stored at $\leq 20^{\circ} \mathrm{C}$. It was thawed immediately before additional investigations. All blood samples were taken from patients during an attack-free interval.

Besides APTT, C1-INH activity and protein concentration, C4 protein concentration, international normalized ratio (INR) based on the PT, clotting activity of FXI and FXII and TAT complex were determined to obtain information on the activation of the kallikrein-kinin (contact) system and coagulation system in order to complement the APTT results.

For APTT determination, PPP was mixed and incubated with nonsettling colloidal silica and synthetic phospolipids [SynthASil ${ }^{\circledR}$, Instrumentation Laboratory (IL), Bedford, Mass., USA] for $180-220 \mathrm{~s}$ at $37^{\circ} \mathrm{C}$. After this, the mixture was recalcified with $0.020 \mathrm{~mol} / \mathrm{l}$ calcium chloride solution, and the clotting time measured. Determination of FXI and FXII was done by a modification of APTT using SynthASil ${ }^{\circledR}$ and FXI- and FXII-deficient plasma (IL), respectively. PT was determined by mixing PPP with a thromboplastin reagent containing recombinant tissue factor, phospholipids and calcium ions (RecombiPlasTin 2G kit, IL) and measuring the clotting time. INR was calculated with the formula: (patient's PT/mean of PT normal range $)^{\mathrm{ISI}}$. APTT, FXI, FXII and PT were determined using an automated coagulation analyzer (ACL TOP 700 CTS, IL).

TAT was determined by ELISA technique (Enzygnost ${ }^{\circledR}$ TAT micro, Siemens Healthcare Diagnostics Product GmbH, Marburg, Germany) [17]. C1-INH activity was measured with the chromogenic substrate method and $\mathrm{C} 1-\mathrm{INH}$ and $\mathrm{C} 4$ protein concentrations by radial immunodiffusion as described previously [18]. 
Table 2. Patients with shortened APTT in various forms of angioedema and HP

\begin{tabular}{llllll}
\hline Diagnosis & $\begin{array}{l}\text { Total number } \\
\text { of patients }\end{array}$ & $\begin{array}{l}\text { Number of } \\
\text { patients with } \\
\text { shortened APTT } \\
(<26 s)^{\mathrm{a}}\end{array}$ & $\begin{array}{l}\text { Percentage of } \\
\text { patients with } \\
\text { shortened APTT } \\
(<26 \mathrm{~s})^{\mathrm{a}}\end{array}$ & $\begin{array}{l}\text { Number of } \\
\text { patients with } \\
\text { shortened APTT } \\
(<24 \mathrm{~s})\end{array}$ & $\begin{array}{l}\text { Percentage of } \\
\text { patients with } \\
\text { shortened APTT } \\
(<24 \mathrm{~s})\end{array}$ \\
\hline HAE-C1-INH type I & 41 & 30 & 73.2 & 13 & 31.7 \\
HAE-C1-INH type II & 4 & 3 & 75 & 1 & 25 \\
HAE-FXII & 13 & 1 & 7.7 & 0 & 0 \\
HAE-unknown & 14 & 2 & 14.3 & 0 & 0 \\
AAE-C1-INH & 15 & 10 & 66.7 & 8 & 53.3 \\
IAE-h & 32 & 1 & 3.1 & 0 & 0 \\
IAE-nh & 30 & 4 & 13.3 & 1 & 3.3 \\
HP & 26 & 2 & 7.7 & 0 & 0 \\
\hline
\end{tabular}

APTT RR: 26-36 s.

a The lower limit of RR.

Statistical Analysis

The results of patients with low C1-INH activity were compared with those patients with normal C1-INH activity and HP.

ANOVA was done by using Smith's Statistical Package v2.80. The patient groups were compared with HP. $\mathrm{p}=0.05$ was statistically significant and $\mathrm{p}=0.001$ was statistically highly significant. The predictive values were calculated by using STAT 507 epidemiological research methods (https://onlinecourses.science.psu. edu/stat507, assessed 18/3/2016). Prior to any study procedures, all patients and HP gave their informed consent and approval for the study was obtained from the local ethics committee in Mainz.

\section{Results}

Compared to HP, mean C1-INH activity and C4 protein concentration were low in HAE-C1-INH types I and II and in AAE-C1-INH (table 1). The mean C1-INH protein concentration was low in HAE-C1-INH type I and AAE-C1-INH and normal in HAE-C1-INH type II. These values were normal in HAEnCI, IAE-h, IAE-nh and HP. Mean APTT was significantly shortened in HAE-C1INH types I and II and AAE-C1-INH and normal in HAEnCI, IAE-h and IAE-nh when compared to HP (table 1). The APTT RR was $26-36$ s. Table 2 shows a shortening of APTT below the RR in $73.2 \%$ of HAE-C1-INH type I patients, $75 \%$ of HAE-C1-INH type II patients, $66.7 \%$ of AAE-C1-INH patients, $7.7 \%$ of HAE-FXII patients, $14.3 \%$ of HAE-unknown patients, $3.1 \%$ of IAE-h patients, $13.3 \%$ of IAE-nh patients and in $9 \%$ of HP. Summarizing both types of HAE-C1-INH, shortened APTT was observed in $33 / 45$ (73.3\%) patients. Summarizing the types of HAEnCI, shortened APTT was observed in 3/27

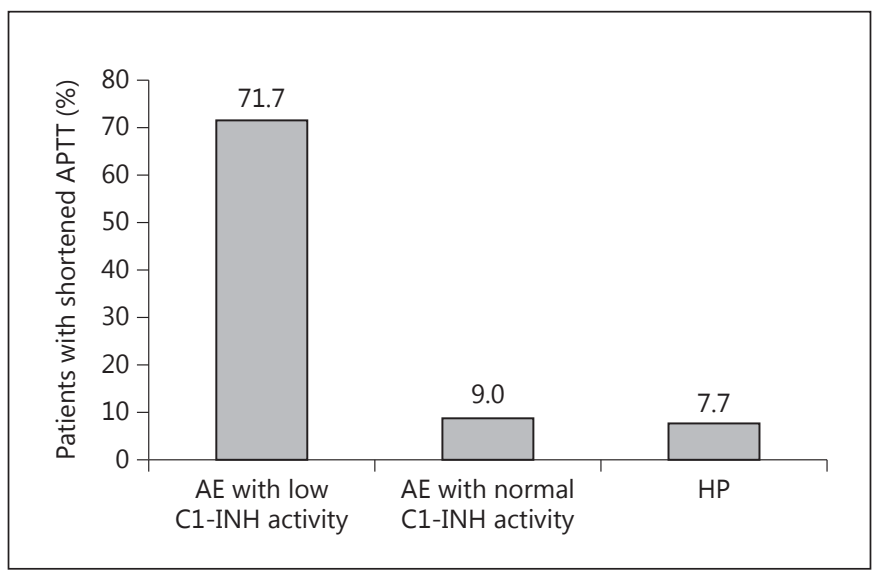

Fig. 2. Percentage of patients with shortened APTT in the HAE/ AAE-C1-INH group, in the group with various forms of angioedema with normal C1-INH and in HP. AE = Patients with angioedema.

(11.1\%) patients. Of the total of 149 patients, 23 (15.4\%) had a particularly strong shortening of the APTT $(<24 \mathrm{~s})$. Of these 23 (96\%), 22 had a C1-INH deficiency; 7 of the $22(31.8 \%)$ with very low C1-INH activity $(\leq 1 \%)$ had a normal APTT. The mean APTT was $25.0 \mathrm{~s}(\mathrm{SD} \pm 2.5)$ for all HAE-C1-INH and AAE-C1-INH patients $(\mathrm{n}=60)$, $29.5 \mathrm{~s}(\mathrm{SD} \pm 2.4)$ for all $\mathrm{HP}(\mathrm{n}=26)$ and $29.3 \mathrm{~s}(\mathrm{SD} \pm 2.8)$ for all HAEnCI/IAE patients $(\mathrm{n}=89)$. The differences between HAE/AAE-C1-INH and HP and HAEnCI/IAE were statistically highly significant $(\mathrm{p}<0.0001)$, but those between HAEnCI/IAE and HP were not $(\mathrm{p}=0.1059)$. Figure 2 shows a shortened APTT in $71.7 \%$, i.e. $8-9$ times 
Table 3. Predictive values for APTT in 149 angioedema patients

\begin{tabular}{|c|c|c|c|c|c|}
\hline & \multicolumn{2}{|c|}{$\begin{array}{l}\text { HAE-C1-INH types I and II, } \\
\text { AAE-C1-INH }\end{array}$} & \multicolumn{2}{|c|}{$\begin{array}{l}\text { HAE-FXII, HAE-unknown, } \\
\text { IAE-h, IAE-nh }\end{array}$} & Total \\
\hline \multirow[t]{2}{*}{ C1-INH activity, \% } & \multicolumn{2}{|l|}{$1-48$} & \multicolumn{3}{|c|}{$55-130$} \\
\hline & Cell & $\mathrm{n}$ & Cell & $\mathrm{n}$ & $\mathrm{n}$ \\
\hline PPV (APTT <26 s) & A & 43 & B & 8 & 51 \\
\hline NPV (APTT $\geq 26 s)$ & $\mathrm{C}$ & 17 & $\mathrm{D}$ & 81 & 98 \\
\hline Total number of patients & 60 & & 89 & & 149 \\
\hline
\end{tabular}

APTT RR: 26-36 s. C1-INH activity (gold standard) RR was between 70 and 130\%. Prevalence of C1-INH deficiency $\left[\mathrm{T}_{\mathrm{Cl} \text {-INH deficiency }} /\right.$ Total $\left.\times 100\right]=40.3 \%$. Cells: $\mathrm{A}=$ true positive; $\mathrm{B}=$ false positive; $\mathrm{C}=$ false negative; $\mathrm{D}=$ true negative. Sensitivity $[\mathrm{A} /(\mathrm{A}+\mathrm{C}) \times 100]=71.7 \%$; specificity $[\mathrm{D} /(\mathrm{D}+\mathrm{B}) \times 100]=91.0 \%$. PPV $(\mathrm{HAE}-\mathrm{FXII}$, HAE-unknown, IAE-h, IAE-nh) $[\mathrm{A} /(\mathrm{A}+\mathrm{B}) \times 100]=84.3 \%$. NPV (HAE-FXII, HAE-unknown, IAE-h, IAE-nh) $[D /(D+C) \times 100]=82.7 \%$.

more, in the angioedema types associated with $\mathrm{C} 1-\mathrm{INH}$ deficiency (HAE/AAE-C1-INH) compared to those with normal C1-INH (9\%) and to HP (7.7\%).

The predictive values of shortened APTTs for all 149 AE patients are presented in table 3. The prevalence of C1-INH deficiency was $40.3 \%$ in this group of patients. The positive predictive value (PPV) was $84.3 \%$ and negative predictive value (NPV) $82.7 \%$. This means that $84.3 \%$ of AE patients with short APTTs will have low C1-INH activity and $82.7 \%$ with normal APTTs will have normal or subnormal C1-INH activity. Changes in prevalence have an influence on PPV and NPV. Increases will increase PPV and decrease NPV and vice versa.

As shown in table 1, the mean FXI clotting activity was significantly increased in HAE-C1-INH type I, AAE-C1INH and IAE-nh, and the mean FXII clotting activity was increased in HAE-C1-INH type 1 and AAE-C1-INH. TAT was significantly increased in HAE-C1-INH type I, HAE-unknown and AAE-C1-INH. None of the 149 angioedema patients showed signs of thromboembolic complications.

\section{Discussion}

Our results show that the APTT is shortened in most HAE-C1-INH type I patients. This is similar to other studies $[5,7,13,16]$ with one exception, where the mean kaolin-cephalin clotting time (equivalent to APTT) was in the upper part of the RR for reasons unknown to us [14]. The majority of our patients with HAE-C1-INH type II and AAE-C1-INH had a shortened APTT, but only a few of those with HAE/AAE with a normal C1-
INH. We therefore assume that a shortened APTT is a consequence of the C1-INH deficiency and a sign of the latent activation of the KKS and the intrinsic clotting system [16]. Interestingly, a significant elevation of mean FXI activity in IAE-nh patients alone seems to not be sufficient to shorten APTT. The FXI elevation combined with a normal FXII activity seems to be a sign for an FXIIa-independent activation of the intrinsic coagulation system. This mechanism has been described for thrombin (FIIa) [19]. As a consequence, the angioedema attacks in IAE-nh patients may not be bradykinin-induced [20]. If proven by further investigation, the isolated elevation of FXI may be an important tool to differentiate between IAE-nh and IAE-h. In IAE-h, the FXI activity was normal in the attack-free interval. The difference between IAE-nh and IAE-n was highly significant $(\mathrm{p}<0.0001)$.

Mean elevated TAT values indicated that higher amounts of FIIa are somehow formed and are inactivated by antithrombin, forming an inactive complex (TAT). The mean TAT values of HAE-C1-INH type I, HAE-unknown and AAE-C1-INH were significantly increased versus $\mathrm{HP}$ and were above the RR. In HAE-C1-INH type I and AAE-C1-INH, this is a consequence of the activation of the KKS and the intrinsic coagulation system. We do not have an explanation for the significant TAT elevation in HAE-unknown. The mean FXI value was elevated (117\%), but this elevation was not statistically significant. The value was still within the RR of $70-120 \%$. This elevation could be a sign for a mild activation of the intrinsic coagulation system; however, based on the FXII and FXI values, the reason could be the formation of thrombin by the extrinsic coagulation system [21]. The results for 
HAE-C1-INH type II patients should not be overrated as they were based on only 4 patients.

There were no signs of thromboembolic complications in our study, in contrast to other studies which found that patients with a short APTT had significantly more thromboembolic events, e.g. venous thrombosis [22]. In our investigation, normal INR values showed that the extrinsic clotting system and the common pathway of coagulation were obviously not impaired in the HAE/ AAE-C1-INH and IAE patients.

According to our results, $28.3 \%$ of the 60 patients with inherited or acquired C1-INH deficiency had a normal APTT despite the deficiency. The reason could be a situation in the plasma that normally leads to a prolongation of APTT. However, we only investigated very few plasma components, not enough to give a plausible explanation for the normal APTT values in these patients.

Together with the clinical picture and the history of the patient, low APTT values may be helpful in the diagnosis of HAE-C1-INH and AAE-C1-INH and in differentiating them from angioedema with normal C1-INH.

Under normal conditions, a shortened APTT can be detected in the hemostasis laboratory with a frequency of 6-9\% [23]. Beside the hemostatic changes in HAE/AAEC1-INH patients, a short APTT can also be linked to sample handling, a hypercoagulable state, increased FVIII, cancer, myocardial infarction, hyperthyroidism, diabetes, sickle cell disease and pregnancy $[22,23]$. There may also be conditions which shorten the APTT and are not yet known. Factors that might normalize or even prolong the APTT include a high hematocrit level, anticoagulation (oral anticoagulants or heparin), antiphospolipid antibodies (especially lupus anticoagulant), coagulation factor deficiencies, sepsis and coagulation factor inhibitors [24]. This limits the usefulness of APTT testing for the early diagnosis of HAE- or AAE-C1-INH. The results of PPV and NPV confirmed the usefulness and also the limitations of APTT for such a diagnosis.

In conclusion, APTT is an in vitro test, covering the KKS and the intrinsic and common coagulation pathways. It is mostly used as a screening test for inherited or acquired factor deficiencies and lupus anticoagulant as well as to monitor anticoagulation with heparin. Shortened APTT values should never be ignored. They may indicate an increased risk of thromboembolic events and can be found in cancer, myocardial infarction, hyperthyroidism, diabetes, sickle cell disease and pregnancy. Now we can add HAE-C1-INH and AAE-C1-INH to this list. The APTT may be helpful in the diagnosis of HAE- and AAE-C1-INH in patients with symptoms, especially if $\mathrm{C} 1-\mathrm{INH}$ and $\mathrm{C} 4$ values are not yet available; however, as with the $\mathrm{C} 4$ value, it cannot supersede the determination of C1-INH activity which is needed for the final confirmation of the diagnosis.

\section{Disclosure Statement}

Konrad Bork is a consultant for CSL Behring and Shire. For Günther Witzke, no conflicts of interest are declared.

\section{References}

1 Dewald G, Bork K: Missense mutations in the coagulation factor XII (Hageman factor) gene in hereditary angioedema with normal $\mathrm{C} 1$ inhibitor. Biochem Biophys Res Commun 2006; 343:1286-1289.

2 Caccia S, Suffritti C, Cicardi M: Pathophysiology of hereditary angioedema. Pediatr Allergy Immunol Pulmonol 2014;27:159-163.

3 Cicardi M, Zanichelli A: Acquired angioedema. Allergy Asthma Clin Immunol 2010;6:14.

4 Bork K, Barnstedt SE, Koch P, Traupe H: Hereditary angioedema with normal C1-inhibitor activity in women. Lancet 2000;356:213217.

5 Bork K, Witzke G, Artmann K, Benes P, Bockers $\mathrm{M}$, Kreuz W: Interaction between C1INA, coagulation, fibrinolysis and kinin system in hereditary angioneurotic edema (HANE) and urticaria. Arch Dermatol Res 1984;276:375-380.
6 Cugno M, Zanichelli A, Bellatorre AG, Griffini S, Cicardi M: Plasma biomarkers of acute attacks in patients with angioedema due to C1-inhibitor deficiency. Allergy 2009;64: 254-257.

7 Cullmann W, Kovary PM, Muller N, Dick W: Complement, coagulation and fibrinolytic parameters in hereditary angioedema (HAE). Clin Exp Immunol 1982;49:618-622.

8 Joseph K, Tuscano TB, Kaplan AP: Studies of the mechanisms of bradykinin generation in hereditary angioedema plasma. Ann Allergy Asthma Immunol 2008;101:279-286.

9 Kodama J, Uchida K, Yoshimura S, Katayama Y, Kushiro H, Yutani C, Funahashi S, Takamiya O, Matsumoto Y, Ando Y, et al: Studies of four Japanese families with hereditary angioneurotic edema: simultaneous activation of plasma protease systems and exogenous triggering stimuli. Blut 1984;49:405-418.
10 Nilsson T, Back O: Elevated plasmin-alpha 2-antiplasmin complex levels in hereditary angioedema: evidence for the in vivo efficiency of the intrinsic fibrinolytic system. Thromb Res 1985;40:817-821.

11 Nielsen EW, Johansen HT, Hogasen K, Wuillemin W, Hack CE, Mollnes TE: Activation of the complement, coagulation, fibrinolytic and kallikrein-kinin systems during attacks of hereditary angioedema. Immunopharmacology 1996;33:359-360.

12 van Geffen M, Cugno M, Lap P, Loof A, Cicardi M, van Heerde W: Alterations of coagulation and fibrinolysis in patients with angioedema due to C1-inhibitor deficiency. Clin Exp Immunol 2012;167:472-478.

13 Relan A, Bakhtiari K, van Amersfoort ES, Meijers JC, Hack CE: Recombinant C1-inhibitor: effects on coagulation and fibrinolysis in patients with hereditary angioedema. BioDrugs 2012;26:43-52. 
14 Sawicka B, Zawisza E, Pezko H: Hemostasis system in patients with Quincke's edema before and after treatment with epsilon-aminocaproic acid. Otolaryngol Pol 1984;38:511515.

15 Schapira M, Silver LD, Scott CF, Schmaier AH, Prograis LJ Jr, Curd JG, Colman RW: Prekallikrein activation and high-molecularweight kininogen consumption in hereditary angioedema. N Engl J Med 1983;308:10501053.

16 Csuka D, Veszeli N, Imreh E, Zotter Z, Skopal J, Prohaszka Z, Varga L, Farkas H: Comprehensive study into the activation of the plasma enzyme systems during attacks of hereditary angioedema due to $\mathrm{C} 1$-inhibitor deficiency. Orphanet J Rare Dis 2015;10:132.
17 Pelzer H, Schwarz A, Heimburger N: Determination of human thrombin-antithrombin III complex in plasma with an enzyme-linked immunosorbent assay. Thromb Haemostasis 1988;59:101-106.

18 Bork K, Kleist R, Hardt J, Witzke G: Kallikrein-kinin system and fibrinolysis in hereditary angioedema due to factor XII gene mutation Thr309Lys. Blood Coagul Fibrinolysis 2009;20:325-332.

19 Walsh PN: Roles of platelets and factor XI in the initiation of blood coagulation by thrombin. Thromb Haemost 2001;86:75-82.

20 Azin GM, Suffritti C, Wu MA, Zanichelli A, Maggioni LM, Cicardi M: High molecular weight kininogen cleavage in idiopathic angioedema. J Allergy Clin Immunol 2015; 135(suppl 1):AB198.
21 Mann KG: Thrombin formation. Chest 2003; 124(suppl 3):4S-10S.

22 Korte W, Clarke S, Lefkowitz JB: Short activated partial thromboplastin times are related to increased thrombin generation and an increased risk for thromboembolism. Am J Clin Pathol 2000;113:123-127.

23 Lippi G, Salvagno GL, Ippolito L, Franchini M, Favaloro EJ: Shortened activated partial thromboplastin time: causes and management. Blood Coagul Fibrinolysis 2010;21: 459-463.

24 Kamal AH, Tefferi A, Pruthi RK: How to interpret and pursue an abnormal prothrombin time, activated partial thromboplastin time, and bleeding time in adults. Mayo Clin Proc 2007;82:864-873. 